\title{
Complete genome sequence of the lytic Pseudomonas fluorescens phage $\varphi$ IBB-PF7A
}

\author{
Sanna Sillankorva ${ }^{1,2}$, Leon D Kluskens ${ }^{1}$, Erika J Lingohr ${ }^{3}$, Andrew M Kropinski ${ }^{3,4}$, Peter Neubauer $^{5}$ and \\ Joana Azeredo ${ }^{1 *}$
}

\begin{abstract}
Background: Phage $\varphi$ IBB-PF7A is a T7-like bacteriophage capable of infecting several Pseudomonas fluorescens dairy isolates and is extremely efficient in lysing this bacterium even when growing in biofilms attached to surfaces. This work describes the complete genome sequence of this phage.

Results: The genome consists of a linear double-stranded DNA of 40,973 bp, with 985 bp long direct terminal repeats and a GC content of approximately $56 \%$. There are 52 open reading frames which occupy $94.6 \%$ of the genome ranging from 137 to 3995 nucleotides. Twenty eight (46.7\%) of the proteins encoded by this virus exhibit sequence similarity to coliphage T7 proteins while 34 (81.0\%) are similar to proteins of Pseudomonas phage gh-1.

Conclusions: That this phage is closely related to Pseudomonas putida phage gh-1 and coliphage T7 places it in the "T7-like viruses" genus of the subfamily Autographivirinae within the family Podoviridae. Compared to the genome of gh-1, the sequence of $\varphi$ IBB-PF7A is longer and contains more genes with unassigned function and lacks a few potentially essential and non-essential $T 7$ genes, such as gene1.1, 3.8, and 7.
\end{abstract}

\section{Background}

Formerly, the phylogenetic and taxonomic classification of bacteriophages (phages) was based on similarities in phage morphology, host range [1], ability to recombine, and also on similar cross-hybridization patterns of their DNAs [2-4]. Today, phage genome sequencing is commonly performed for classification and characterization purposes and is based on the arrangement of conserved genes and the nucleotide and protein sequence identity [5,6]. A specific group of phages has a number of conserved proteins but also a relatively high number of proteins for which no match occurs in the current databases. This reveals the great genetic diversity existent within phages. Of the total global estimated phage population $\left(>10^{31}\right)$, only a small number have been completely sequenced.

In the last few years, a large number of T7-like phages have been sequenced most of which have been gathered in a new viral subfamily within the family Podoviridae.

\footnotetext{
* Correspondence: jazeredo@deb.uminho.pt

'IBB-Institute for Biotechnology and Bioengineering, Centre of Biological Engineering, Universidade do Minho, Campus de Gualtar 4710-057, Braga, Portugal

Full list of author information is available at the end of the article
}

The Autographivirinae was formerly referred to as the T7 supergroup of phages [7]. All these phages, most of which have members of the Enterobacteriaceae as their host, present a highly conserved genome organization and mainly differ at sequence level through the presence or absence of nonessential genes [8]. Phages presenting more similarities to each other are grouped in genera within the Autographivirinae, namely the "T7-like viruses", the "SP6-like viruses" and the "phiKMV-like viruses". The genome of coliphage T7 has 56 genes encoding potential proteins [8], of which 35 have known function or functions. In addition, there are 25 nonessential proteins, of which only 12 are conserved across the genus "T7-like viruses" [8].

In this manuscript we report on the full genomic characterization of Pseudomonas fluorescens phage $\varphi$ IBB-PF7A, a phage capable of infecting several dairy P. fluorescens isolates including isolates belonging to different ribotypes [9]. On the basis of TEM micrographs this virus resembled T7-like phages with a head diameter of about $63 \mathrm{~nm}$ and a noncontractile tail size approximately $13 \times 8 \mathrm{~nm}$. In terms of growth cycle, phage $\varphi$ IBB-PF7A has a latent period of 15 minutes, an eclipse period of 10 minutes and a burst size of 153
C Biomed Central

(c) 2011 Sillankorva et al; licensee BioMed Central Ltd. This is an Open Access article distributed under the terms of the Creative Commons Attribution License (http://creativecommons.org/licenses/by/2.0), which permits unrestricted use, distribution, and reproduction in any medium, provided the original work is properly cited. 
plaque forming units per infected cell. Furthermore, this phage has been the focus of several recent studies lysing efficiently its host present in single [10] and dual species biofilms [11]. Moreover, $\varphi$ IBB-PF7A is the first reported phage capable of efficiently infecting elongated $P$. fluorescens cells - which are up to 10 times longer than normal sized cells - and killing planktonic stationary (3 days old) phase cells [9]. For these reasons, a more detailed characterization was desired in order to further increase our knowledge about this particular lytic phage.

\section{Results and Discussion}

This work focuses on the determination of the complete genome sequence of the $P$. fluorescens phage $\varphi$ IBBPF7A. This virus is the first T7-like bacteriophage for $P$. fluorescens for which the genome has been determined.

\section{$\varphi$ IBB-PF7A nucleotide sequence and general sequence properties}

The DNA sequence of phage $\varphi$ IBB-PF7A was determined and it consists of linear double-stranded DNA of $40,973 \mathrm{bp}$. The size of this phage correlates well with other T7-like phage members, where the smallest and largest phages reported so far are the Pseudomonas putida phage gh-1 $(37.4 \mathrm{~kb})$ and the Vibrio parahaemolyticus phage VpV262 (45.9 kb), respectively [12]. The redundant terminal repeats (DTR) of $\varphi$ IBB-PF7A are unusually long (985 bp) for member of the T7 group (T7 has DTRs of only $160 \mathrm{bp}$ [13]) and, up till now, the genome of $P$. aeruginosa phage LKD16 had the longest DTRs (428 bp) [14]. Phage $\varphi$ IBB-PF7A has an overall genomic guanine plus cytosine (GC) content of $56.3 \%$, well within the range of GC contents observed in other T7-like phages (46.2 - 62.3\%) [15].

The genome of $\varphi$ IBB-PF7A was scanned for open reading frames (ORFs) of $100 \mathrm{bp}$ or longer using computational software complemented with visual inspection of the genome sequence. The search resulted in 52 predicted genes with lengths ranging from 137 to 3995 nt. The initiation codon of $92 \%$ of the protein-coding genes is ATG and only four other ORFs, including RNA polymerase (gene1) and the packaging protein $B$ (gene19), start with GTG. The temporal and functional distributions of genes are tightly organized and packed close to each other so that they occupy $94.6 \%$ of the entire nucleotide sequence.

\section{Comparative genomics of phage $\varphi$ IBB-PF7A}

All predicted protein-coding genes were screening using BLASTP against the non-redundant protein database at NCBI. From the 52 ORFs of $\varphi$ IBB-PF7A: i) 25 (48.1\%) have assigned function; ii) $12(23.1 \%)$ are similar to proteins of unknown function in the nonredundant databases and (iii) 15 (28.8\%) code for hypothetical proteins unique to this bacteriophage. While the great majority (34) of the homologs is to proteins of Pseudomonas phage gh-1, examples of primary sequence similarity to Pseudomonas phages phi-2 and LKA1, coliphage T3, Yersinia phage $\phi$ YeO3-12, Vibrio phage ICP3_2008_A and Kluyvera phage Kvp1 exist. With the exceptions of Pseudomonas phage LKA1 which is a member of the "phiKMV-like viruses," all of these phages are members of the "T7-like viruses" genus. Since 28 gene products were related to $\mathrm{T} 7$ phage proteins, the T7 gene nomenclature was adopted for these genes (Additional file 1, Table S1). No function can be speculated about the hypothetical proteins of phage $\varphi$ IBB-PF7A without further study. Based upon overall protein homology determined using CoreGenes [16,17] this virus shares 28 (47\%), 11 (21\%) and 10 (20\%), similar proteins with phages T7, SP6 and $\phi \mathrm{KMV}$, respectively. These results indicate that it is a member of the Autographivirinae, specifically a member of the "T7-like viruses" genus [7].

At the protein level $\varphi$ IBB-PF7A showed the greatest sequence identity with proteins of phage gh-1, and therefore the gene clustering of the two phages was compared (Figure 1). Unlike $P$. putida phage gh-1, which presents no putative genes before gene 1 , in $\varphi$ IBB-PF7A we observed six unique hypothetical proteins of which two (orf1 and orf2) are part of the 985 bp long DTR. Furthermore, these first six ORFs do not display sequence similarity to any of the $\mathrm{T} 7$ genes between gene 0.3 and

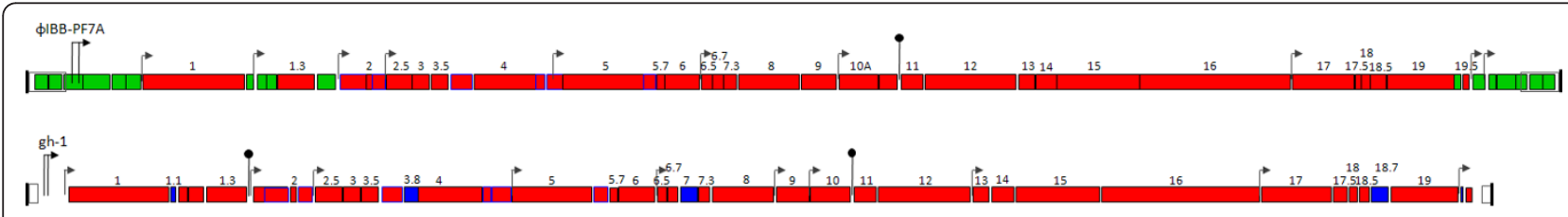

Figure 1 Genomic map of $\boldsymbol{P}$. fluorescens phage $\boldsymbol{\varphi}$ IBB-PF7A and $\boldsymbol{P}$. putida phage gh-1. Filled green boxes are hypothetical proteins unique of $\varphi$ IBB-PF7A, red filled boxes with black and blue outlines indicate genes of $\varphi$ IBB-PF7A which show homology to conserved essential genes of and to nonessential conserved genes of gh-1, blue filled boxes are genes present only in phage gh-1. Putative transcription promoters are indicated by lines with arrowhead: black - early host RNA polymerase promoters; dark grey - phage RNA promoters. Lines with filled black spheres indicate $\rho$-independent terminators. Unfilled dark grey outlined boxes indicate direct terminal repeats and dark lines at left and right indicate the ends of the genome. 
gene0.7. Compared to gh-1, phage $\varphi$ IBB-PF7A also lacks gene 1.1, a gene with unknown function.

Despite the different host ranges of $P$. fluorescens phage $\varphi$ IBB-PF7A and $P$. putida phage gh- 1 , there are 34 phage proteins which show significant sequence identity (Figure 1). As pointed out above, phage gh-1 belongs to the same viral genus as $\varphi$ IBB-PF7A and its sequence is linear double-stranded DNA of 37,359 bp with 216 bp direct terminal repeats, and has 43 predicted putative genes.

A Mauve alignment $[16,18]$ of phage $\varphi$ IBB-PF7A DNA against the genomes of gh- $1, \mathrm{~T} 3$ and $\mathrm{T} 7$ show the regions of DNA sequence similarity (colored areas) interspersed with regions (white) where no sequence similarity exists (Additional file 2; Figure S1).

\section{DNA penetration}

The genes essential of forming the channel which allows the phage DNA injection into the hosts' cytoplasm are all present in phage $\varphi$ IBB-PF7A, namely gene14, gene15 and gene16.

\section{$\varphi$ IBB-PF7A gene arrangement}

\section{A. Early gene expression}

The early genes of this type of virus are transcribed by the host RNA polymerase. The transcribed genes include genes which function to overcome host restriction and to convert the metabolism of the host cell to the production of phage proteins, e.g. synthesis of the phage promoter-specific RNA polymerase (RNAP; gene1). Two host-type promoters were discovered (Additional file 3; Table S2). Interestingly, the early region of this phage contains six ORFs, two of which are in the LTR, which show no sequence similarity at the DNA or protein level to the early genes of any other member of the Autographivirinae. Indeed, this is the first report for the existence of putative ORFs within the terminal repeats of a member of this subfamily, though they are found in phages such as T5 and SPO1. $\varphi$ IBB-PF7A does not encode the T7-like Gp0.3 (ocr), 0.6 or 0.7 homologs.

\section{B. Middle gene expression}

The class II genes are transcribed by the phage RNAP and are involved in DNA replication. Both phages $\varphi$ IBBPF7A and gh-1 lack several class II genes present in T7, namely: gene1.4, gene1.5, gene1.6, gene1.7, gene1.8, gene3.2, gene3.7, gene4.3, and gene5.5. Most of these class II genes are nonessential and only gene1.7 is considered an essential gene and reportedly is beneficial for phage growth [8]. Besides the genes not present in both $\varphi$ IBB-PF7A and gh-1, $\varphi$ IBB-PF7A is also missing the nonessential homing endonuclease (gene3.8).

\section{Late gene expression}

The late genes or class III genes are transcribed by the phage RNAP and are mostly involved in morphogenesis and host lysis. The majority of putative genes found in
T7-like phages are also present in $\varphi$ IBB-PF7A (Additional file 1, Table S1). Phage $\varphi$ IBB-PF7A is only missing gene7, a nonessential protein which is usually involved in host range [8]. Compared to gh-1, phage $\varphi$ IBB-PF7A has more ORFs situated just before the right DTR (Figure 1). We have identified two ORFs which display sequence similarity to the major capsid proteins of phages belonging to the "T7-like viruses." The upstream gene displays homology to the capsid gene of Yersinia phage $\phi \mathrm{YeO} 3-$ 12 while the downstream gene is similar to the analogous protein in P. putida phage gh-1. That these two genes might be coexpressed as a single fusion protein is suggested by the presence of a slippery site (GTTATCGAAAAGGCGTAA) which is translated as VIEKA(stop); but could be extended, via -1 frameshifting, into VIEKgvsvpdp...(stop). While a diagnostic pseudoknot was discovered immediately downstream of the potential slippery site [19-22] there is no proteomic evidence for frameshifting in this bacteriophage [9]. The structural proteome of this phage is therefore made up of gene10A (capsid), the connector gene8, and a conspicuous core made by the proteins gene14, gene15, and gene16 [23].

Phage $\varphi$ IBB-PF7A contains gene16, the internal virion protein $\mathrm{D}$, which contains peptidoglycan hydrolase motif. This activity is essential for enlarging a hole across the cell wall of cells when hosts are infected at low temperatures [8]. Possibly, due to the presence of this protein phage $\varphi$ IBB-PF7A showed a high efficiency towards mature biofilms [10].

\section{Lysis genes}

Endolysins are muralytic enzymes produced by dsDNA phages which hydrolyze the peptidoglycan layer of bacterial cell walls. As in T7 phages, gene3.5 of $\varphi$ IBB-PF7A is proposed to be the endolysin-like protein which possesses $\mathrm{N}$-acetylmuramoyl-L-alanine amidase activity, but is apparently not essential for lysis (I. Molineux, personal communication). The access of endolysins to the cell wall occurs through the presence of a secondary lysis factor known as holin. The small membrane protein derived from gene17.5 of $\varphi$ IBB-PF7A is proposed as holin and it showed highest sequence similarity to Morganella phage MmP1 lysis protein. Phage $\varphi$ IBB-PF7A has also one more lysis gene, the $\lambda \mathrm{Rz}$-like lysis protein (gene18.5) which presents a $34 \%$ sequence identity to the homologous protein in phage gh- 1 . The proteins of phage $\varphi$ IBB-PF7A was scanned for transmembrane helices using TMHMM. A total of six ORFS contain transmembrane helices (TMD, orf2, gene17.5, gene18.5, gene19, and gene19.5, and orf52), with only one (gene17.5) presenting two TMD.

\section{Putative regulatory elements}

No tRNA genes were predicted in the $\varphi I B B-P F 7 A$ genome using tRNAscan-SE. Several potential putative 
promoters were identified in the DNA sequence of $\varphi$ IBB-PF7A, using PHIRE and BPROM (Additional file 3 , Table S2). Using strict criteria for the assignment of host promoter sites, i.e. that four out of the six bp in the -10 and -35 regions be conserved, two host-dependent promoters were discovered. These are both located in orf4. We have been unable to definitively assign promoters to earlier ORFs.

In all $\mathrm{T} 7$ group phages, the phage RNA polymerase (RNAP) is responsible for the recognition of phagespecific promoters. In phage $\varphi$ IBB-PF7A, we identified 12 phage RNAP specific promoters (Additional file 3, Table S2) which are named according to the downstream gene. Eleven promoters were identified which show remarkable sequence conservation with a consensus of taAAAtmCCCTCACCwrAAcAGGGa (Figure 2). Interestingly the consensus sequence is 1 bp longer than that for the other members of this genus.

The promoter sequences all lie in intergenic regions and show the greatest sequence similarity to those of coliphage T3 (Additional file 3, Table S2). The specificity of the RNAP is determined by the positions -10 and -11 and it is the position -2 that establishes the promoter strength [24]. The highest relative utilization of the promoter is observed when there is a $T$ at the -2 position. Phage $\varphi$ IBB-PF7A may have lower promoter utilization since there is not a $\mathrm{T}$ positioned at -2 . Nonetheless, the promoter positions -10 and -11 are identical to the consensus sequence of T3 and SP6 (C s, respectively). In phage $\varphi$ IBB-PF7A, seven of the promoters were found to be present in identical places as in phage T7: $\varphi 1, \varphi 2.5, \varphi 6.5, \varphi 9, \varphi 10 \mathrm{~A}, \varphi 17$ and $\varphi 19.5$, respectively. The promoter sequences which were found in analogous genomic positions to the ones in T7 suggest that the regulation of phage mRNA synthesis is well conserved within the group.

FindTerm, RibEx and TransTerm programs were used to search for transcriptional terminators. T7 and gh-1

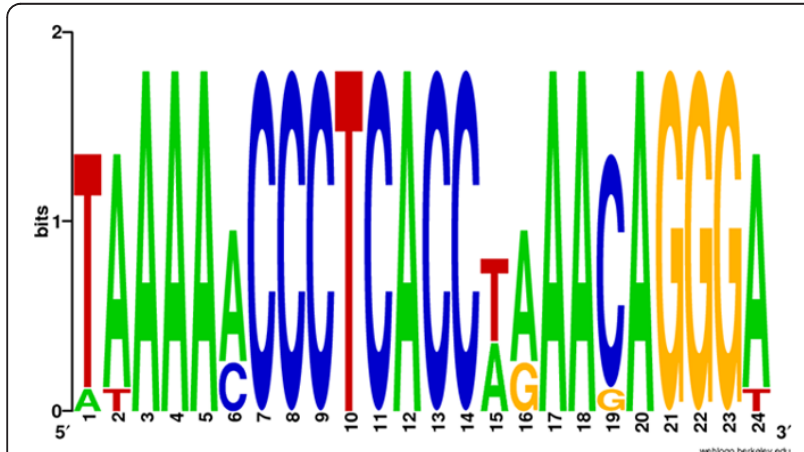

Figure 2 WebLogo of the phage specific promoter sequences of $\boldsymbol{\varphi}$ IBB-PF7A. WebLogo was created at http://weblogo.berkeley. edu/logo.cgi. phages have three terminators identified while phage $\varphi$ IBB-PF7A has only one rho-independent terminator downstream of gene10 (22995 to 23040) which suggest the presence of the Tø2 terminators (named $\mathrm{T}_{\text {Late } 1}$ in phage gh-1). Downstream of gene1.3 is a sequence (gcctgtaccttgcagggc) which exhibits a $\Delta \mathrm{G}$ of $-11.60 \mathrm{kcal} /$ mol. While this sequence is identically placed to the rhoindependent terminator of most T7-like phages it lacks the classical polyT tail.

Summarizing, the genome sequencing of $\varphi$ IBB-PF7A showed similarity to "T7-like viruses" of the subfamily Autographivirinae and allowed the recognition of a new subgroup consisting, so far, of only phages $\varphi$ IBB-PF7A and gh-1.

\section{Methods}

\section{Bacteria and bacteriophage $\varphi$ IBB-PF7A}

The bacterium used (P. fluorescens PF7) was isolated from a dairy industry (Estação Experimental, Paços de Ferreira, Portugal), identified using the API 20NE panel (BioMérieux S.A., France), and grown at $30^{\circ} \mathrm{C}$ in Tryptic Soy Broth (TSB, Fluka) or TSB agar plates for bacteriophage propagation. Bacteriophage $\varphi$ IBB-PF7A was isolated from raw sewage and has been previously characterized according to morphology, growth cycle and other parameters [9].

\section{Bacteriophage $\varphi$ IBB-PF7A propagation}

For phage amplification, the plate lysis and elution method described by Sambrook \& Russell [25] was used with some modifications. Briefly, $1 \mathrm{ml}$ of phage $\left(1 \times 10^{3}\right.$ PFU ml $\mathrm{m}^{-1}$ ) and $1 \mathrm{ml}$ of overnight grown host were mixed with $30 \mathrm{ml}$ of TSB soft agar (TSB $+0.6 \%$ agar) and added to $250 \mathrm{ml} \mathrm{T}$-flasks with a thin layer of TSA media. The soft-agar layer was allowed to solidify and the flasks were incubated at $30^{\circ} \mathrm{C}$ for $7 \mathrm{~h}$. The phage particles were subsequently eluted with SM buffer and concentrated with PEG 8000, and then extracted with chloroform. Samples in $\mathrm{SM}$ buffer were stored at $4^{\circ} \mathrm{C}$ until further use.

\section{DNA isolation}

Phage DNA was isolated using a Wizard Lambda Preps DNA purification system (Promega, Madison, WI.) according to the manufacturer's instructions.

\section{Phage DNA sequencing}

Phage $\varphi$ IBB-PF7A DNA was sheared with a nebulizer. Following 1\% agarose gel electrophoresis overnight electrophoresis at $30 \mathrm{~V}$, fractions with sizes between 1 and $4 \mathrm{~kb}$ were cut from the gels and the DNA purified. The ends were repaired, the fragments were ligated overnight, at $16^{\circ} \mathrm{C}$, into pZero cloning vector (Zero background/Kan cloning kit, Invitrogen, Carlsbad, CA) previously digested with EcoRV, and transformed into 
chemically competent One Shot ${ }^{\mathbb{B}}$ TOP10 cells (Invitrogen). The cells were recovered in SOC medium, incubated for $1 \mathrm{~h}$ at $37^{\circ} \mathrm{C}$ and $225 \mathrm{rpm}$ (Titramax plate shaker, Heidolph Elektro GmbH \& Co. KG, Kelheim, Germany) and plated onto LB-Kan plates containing XGal. Selected clones were picked with a Genetix QPix2 robotic colony picker (Genetix Corp., Boston, MA) and grown overnight in $\mathrm{LB}$ medium at $37^{\circ} \mathrm{C}$ and $225 \mathrm{rpm}$. The cultures were centrifuged and plasmid DNA was extracted. For sequencing, Big Dye chemistry was used and the sequencing was carried out in ABI 3700 DNA Sequencer (Applied Biosystems). Sequence reads were assembled using GAP4 (Staden package software) and the sequences were assembled into contigs [26].

Several frameshift errors were corrected, through a primer-walking technique with 20-mer oligonucleotide primers and pure $\varphi$ IBB-PF7A DNA with the amplicons being sequenced.

\section{ORF prediction and annotation}

Prediction of open reading frames (ORFs) was performed using GeneMark.hmm, OrfFinder and Kodon (Applied Maths, Austin, TX). For tRNA gene identification, the tRNAscan-SE program was used [27].

Translated ORFs were compared with known protein sequences using BLASTP, against the non-redundant protein GenBank database. To tease out function conserved hypothetical proteins were also analyzed by HHPred at http://toolkit.tuebingen.mpg.de/hhpred.

Molecular masses and isoelectric points of phage $\varphi$ IBBPF7A translated putative proteins were determined using the ExPASy Compute pI/Mw tool http://au.expasy.org/ tools/pi_tool.html. Promoter predictions were made using promoter predictor http://www.fruitfly.org/seq_tools/promoter.html, PHIRE 1.0 [28] and BPROM http:// linux1.softberry.com/berry.phtml?topic=bprom\&group=programs\&subgroup=gfindb and the search for transmembrane helices was done by using TMHMM http:// www.cbs.dtu.dk/services/TMHMM-2.0/. Terminators were predicted using FindTerm http://linux1.softberry. com/berry.phtml?topic=findterm\&group=programs\&subgroup=gfindb, RibEx http://132.248.32.45:8080/cgi-bin/ ribex.cgi and TransTerm http://nbc11.biologie.uni-kl.de/ framed/left/menu/auto/right/transterm/.

\section{Nucleotide sequence accession number}

The GenBank accession number of the complete genomic sequence of phage $\varphi$ IBB-PF7A is GU583987.

\section{Additional material}

Additional file 1: Table S1 Features of phage $\phi$ IBB-PF7A open reading frames and their homology to other phage proteins. Supplementary table
Additional file 2: Figure S1 Figure showing progressive Mauve alignment of the genomes of $P$. fluorescens phage $\varphi$ IBB-PF7A, $P$. putida phage gh-1, coliphages T3 and T7. Supplementary figure.

Additional file 3: Table S2 Predicted promoter sequences using PHIRE and BPROM. Supplementary table

\section{Acknowledgements}

SS acknowledges the financial support from the Portuguese Foundation for Science and Technology (FCT) (grant SFRH/BD/18485/2004). AK acknowledges the financial support of a discovery grant from the Natural Sciences and Engineering Research Council of Canada.

\section{Author details}

'IBB-Institute for Biotechnology and Bioengineering, Centre of Biological Engineering, Universidade do Minho, Campus de Gualtar 4710-057, Braga, Portugal. ${ }^{2}$ Bioprocess Engineering Laboratory, Department of Process and Environmental Engineering and Biocenter Oulu, P.O. Box 4300, FIN-90014 University of Oulu, Finland. ${ }^{3}$ Public Health Agency of Canada, Laboratory for Foodborne Zoonoses, Guelph, Ontario N1G 3W4, Canada. ${ }^{4}$ Department of Molecular and Cellular Biology, University of Guelph, Guelph, Ontario N1G 2W1, Canada. ${ }^{5}$ Department of Biotechnology, Technische Universität Berlin, Berlin, Germany.

\section{Authors' contributions}

SS performed the experiments, annotated the genome and prepared the manuscript, LDK and AMK contributed to the genome annotation; AMK also contributed to manuscript preparation; EJL corrected the errors in the genome sequence; JA and PN supervised the work. The final manuscript was read and accepted by all co-authors.

\section{Competing interests}

The authors declare that they have no competing interests.

Received: 26 July 2010 Accepted: 26 March 2011

Published: 26 March 2011

\section{References}

1. Adams MH: Bacteriophages. New York: Interscience Publishers; 1959.

2. Botstein D: A theory of modular evolution for bacteriophages. Ann N Y Acad Sci 1980, 354:484-490.

3. Campbell A: Comparative molecular biology of lambdoid phages. Annu Rev Microbiol 1994, 48:193-222.

4. Casjens S, Wyckoff E, Hayden M: Bacteriophage P22 portal protein is part of the gauge that regulates packing density of intravirion DNA. Journal of Molecular Biology 1992, 224:1055-1074.

5. Brüssow H, Desiere F: Comparative phage genomics and the evolution of Siphoviridae: insights from dairy phages. Mol Microbiol 2001, 39:213-222.

6. Lucchini S, Desiere F, Brüssow H: Comparative genomics of Streptococcus thermophilus phage species supports a modular evolution theory. J Virol 1999, 73:8647-8656.

7. Lavigne R, Seto D, Mahadevan P, Ackermann HW, Kropinski AM: Unifying classical and molecular taxonomic classification: analysis of the Podoviridae using BLASTP-based tools. Research in Microbiology 2008, 159:406-414.

8. Molineux IJ: The T7 Group. In The bacteriophages. Edited by: Calendar RL. Oxford: Oxford University Press; 2005.

9. Sillankorva S, Neubauer P, Azeredo J: Isolation and characterization of a T7like lytic phage for Pseudomonas fluorescens. Bmc Biotechnology 2008, 8:80.

10. Sillankorva S, Neubauer P, Azeredo J: Pseudomonas fluorescens biofilms subjected to phage IBB-PF7A. Bmc Biotechnology 2008, 8:79.

11. Sillankorva S, Neubauer P, Azeredo J: Phage control of dual species biofilms of Pseudomonas fluorescens and Staphylococcus lentus. Biofouling 2008.

12. Kovalyova IV, Kropinski AM: The complete genomic sequence of lytic bacteriophage gh-1 infecting Pseudomonas putida-evidence for close relationship to the T7 group. Virology 2003, 311:305-315

13. Dunn JJ, Studier FW: Complete nucleotide sequence of bacteriophage T7 DNA and the locations of T7 genetic elements. Journal of Molecular Biology 1983, 166:477-535. 
14. Ceyssens P-J, Lavigne R, Mattheus W, Chibeu A, Hertveldt K, Mast J, Robben J, Volckaert G: Genomic Analysis of Pseudomonas aeruginosa Phages LKD16 and LKA1: Establishment of the KMV Subgroup within the T7 Supergroup. Journal of Bacteriology 2008, 188:6924-6931.

15. Lavigne R, Burkal'tseva MV, Robben J, Sykilinda NN, Kurochkina LP, Grymonprez B, Jonckx B, Krylov VN, Mesyanzhinov W, Volckaert G: The genome of bacteriophage $\mathbb{K K M V}$, a T7-like virus infecting Pseudomonas aeruginosa. Virology 2008, 312:49-59.

16. Kropinski AM, Borodovsky M, Carver TJ, Cerdeño-Tárraga AM, Darling A, Lomsadze A, Mahadevan P, Stothard P, Seto D, Van Domselaar G, Wishart DS: In silico identification of genes in bacteriophage DNA. Methods Mol Biol 2009, 502:89.

17. Zafar N, Mazumder R, Seto D: CoreGenes: a computational tool for identifying and cataloging "core" genes in a set of small genomes. BMC Bioinformatics 2002, 24:3-12.

18. Darling AC, Mau B, Blattner FR, Perna NT: Mauve: multiple alignment of conserved genomic sequence with rearrangements. Genome Res 2004, 14:1394-1403.

19. Alam SL, Atkins JF, Gesteland RF: Programmed ribosomal frameshifting: Much ado about knotting! Proceedings of the National Academy of Sciences of the United States of America 1999, 96:14177-14179.

20. Farabaugh PJ: Programmed translational frameshifting. Annual Review of Genetics 1996, 30:507-528.

21. Harger JW, Meskauskas A, Dinman JD: An 'integrated model' of programmed ribosomal frameshifting. Trends in Biochemical Sciences 2002, 27:448-454.

22. Reeder J, Steffen P, Giegerich R: pknotsRG: RNA pseudoknot folding including near-optimal structures and sliding windows. Nucleic Acids Research 2007, 35:W320-W324.

23. Garcia $L R$, Molineux IJ: Transcription-independent DNA translocation of bacteriophage T7 DNA into Escherichia coli. Journal of Bacteriology 1996, 178:6921-6929.

24. Klement JF, Moorefield MB, Jorgensen E, Brown JE, Risman S, McAllister WT: Discrimination between bacteriophage $\mathrm{T} 3$ and $\mathrm{T} 7$ promoters by the $\mathrm{T} 3$ and T7 RNA polymerases depends primarily upon a three base-pair region located 10 to 12 base-pairs upstream from the start site. Molecular Biology 1990, 215:21-29.

25. Sambrook J, Russell DW: Molecular Cloning: A Laboratory Manual. New York: Cold Spring Harbor Laboratory Press, Cold Spring Harbor; 2001.

26. Staden R, Beal KF, Bonfield JK: The Staden package, 1998. Methods Mol Biol $2000,132: 130$

27. Lowe TM, Eddy SR: tRNAscan-SE: a program for improved detection of transfer RNA genes in genomic sequence. Nucleic Acids Res 1997, 1(25):955-964.

28. Lavigne R, Sun WD, Volckaert G: PHIRE, a deterministic approach to reveal regulatory elements in bacteriophage genomes. Bioinformatics 2004 20:629-U99.

doi:10.1186/1743-422X-8-142

Cite this article as: Sillankorva et al: Complete genome sequence of the lytic Pseudomonas fluorescens phage pIBB-PF7A. Virology Journal 2011 8:142.

\section{Submit your next manuscript to BioMed Central and take full advantage of:}

- Convenient online submission

- Thorough peer review

- No space constraints or color figure charges

- Immediate publication on acceptance

- Inclusion in PubMed, CAS, Scopus and Google Scholar

- Research which is freely available for redistribution

Submit your manuscript at www.biomedcentral.com/submit
Biomed Central 